\title{
Full genome SNP-based phylogenetic analysis reveals the origin and global spread of Brucella melitensis
}

Kim-Kee Tan ${ }^{1,2}$, Yung-Chie Tan ${ }^{3}$, Li-Yen Chang ${ }^{1,2}$, Kok Wei Lee ${ }^{3}$, Siti Sarah Nore', Wai-Yan Yee ${ }^{3}$, Mohd Noor Mat Isa ${ }^{4}$, Faizatul Lela Jafar ${ }^{2}$, Chee-Choong Hoh ${ }^{3}$ and Sazaly AbuBakar ${ }^{1,2^{*}}$

\begin{abstract}
Background: Brucellosis is an important zoonotic disease that affects both humans and animals. We sequenced the full genome and characterised the genetic diversity of two Brucella melitensis isolates from Malaysia and the Philippines. In addition, we performed a comparative whole-genome single nucleotide polymorphism (SNP) analysis of $B$. melitensis strains collected from around the world, to investigate the potential origin and the history of the global spread of B. melitensis.

Results: Single sequencing runs of each genome resulted in draft genome sequences of MY1483/09 and Phil1136/12, which covered $99.85 \%$ and $99.92 \%$ of the complete genome sequences, respectively. The B. melitensis genome sequences, and two B. abortus strains used as the outgroup strains, yielded a total of 13,728 SNP sites. Phylogenetic analysis using whole-genome SNPs and geographical distribution of the isolates revealed spatial clustering of the B. melitensis isolates into five genotypes, I, II, III, IV and V. The Mediterranean strains, identified as genotype I, occupied the basal node of the phylogenetic tree, suggesting that $B$. melitensis may have originated from the Mediterranean regions. All of the Asian $B$. melitensis strains clustered into genotype II with the SEA strains, including the two isolates sequenced in this study, forming a distinct clade denoted here as genotype IId. Genotypes III, IV and V of B. melitensis demonstrated a restricted geographical distribution, with genotype III representing the African lineage, genotype IV representing the European lineage and genotype $V$ representing the American lineage.

Conclusion: We showed that SNPs retrieved from the B. melitensis draft full genomes were sufficient to resolve the interspecies relationships between $B$. melitensis strains and to discriminate between the vaccine and endemic strains. Phylogeographic reconstruction of the history of $B$. melitensis global spread at a finer scale by using whole-genome SNP analyses supported the origin of all $B$. melitensis strains from the Mediterranean region. The possible global distribution of B. melitensis following the ancient trade routes was also consistent with whole-genome SNP phylogeny. The whole genome SNP phylogenetics analysis, hence is a powerful tool for intraspecies discrimination of closely related species.
\end{abstract}

Keywords: Brucella, Phylogenetic, Genome, SNPs, Zoonotic, Bioterrorism

\footnotetext{
* Correspondence: sazaly@um.edu.my

${ }^{1}$ Tropical Infectious Diseases Research and Education Centre (TIDREC),

University of Malaya, 50603 Kuala Lumpur, Malaysia

${ }^{2}$ Department of Medical Microbiology, Faculty of Medicine, University of

Malaya, 50603 Kuala Lumpur, Malaysia

Full list of author information is available at the end of the article
} 


\section{Background}

Brucella spp. are listed as a category B potential biological warfare agent [1]. A low infectious dose of 100 to 1,000 organisms is sufficient to cause an infection. The mechanism of transmission, through aerosols or food chains, makes them easily transmissible to both humans and animals. Extensive studies have been done in the past exploring the potential of Brucella spp. as candidate biological weapon agents [2]. A biological warfare attack model built in 1997, based on an aerosol attack using B. melitensis in an urban population, was estimated could cause an economic loss of $\$ 477.7$ million per 100,000 persons exposed [3]. Owing to the potentially insidious nature of a bioterrorism attack, the intentional release of any biological warfare agent could be difficult to detect. Tracking unusual strains in a particular region could serve as the first line of defence. Rapid detection of potential bioterrorism activities that usually starts with few indistinguishable cases from a natural outbreak, however, is difficult. It relies heavily on precise and sensitive methods, as well as the availability of information about the organism worldwide. Sporadic outbreaks of brucellosis occur worldwide, the probable geographical origin of the source of infection, however, is difficult to determine.

Current approaches to study B. melitensis genome are often limited to specifically targeted genes, for example, multi-locus variable-number tandem repeat (MLVA) analysis, multi-locus sequencing (MLS) and single nucleotide polymorphisms (SNPs) analysis are insensitive to detect new mutations from new clades and therefore, are not very useful $[4,5]$. Complete genome sequencing is the ideal. The methods for achieving it, however, were previously laborious, expensive and usually required time-consuming gap closing of genome assemblies. It was therefore only used for in-depth evolutionary analysis or screening of potentially useful SNPs or loci to develop assays such as the MLVA, MLS, multiple real time and gene sequencing [6]. With the development of next-generation sequencing (NGS) technologies, full bacterial genome sequencing has become highly accessible [4,7-11]. High quality draft bacterial genomes can be rapidly obtained. Unlike the taxonomically informative or canonical SNP-based approaches, whole-genome sequencing serves as a robust and unbiased method to resolve intraspecies relationships for closely related species such as Brucella spp. [12,13] and Bacillus anthracis [14,15].

To date, there are only a limited number of full genome sequences of $B$. melitensis isolates from different geographical locations available in publicly accessible databases. Only one draft full genome sequence of $B$. melitensis isolated from the Southeast Asia (SEA) region is available [16]. We identified and sequenced two human B. melitensis strains, MY1483/09 and Phil1136/12, which originated from Malaysia and the Philippines, respectively. We demonstrate here that a SNP-based phylogenetic analysis of the draft genome sequence obtained from a single sequencing run was sufficient to resolve the intraspecies genetic relationships of geographically distinct $B$. melitensis strains. In addition, whole-genome-based molecular epidemiology tracking allows the elucidation of the potential origins and spread of B. melitensis.

\section{Results}

\section{General features}

The full genome sequences of the Brucella isolates were generated from a single sequencing run using the 316 chips of the Life Technologies Ion Torrent PGM platform. The resulting reads were de novo assembled into two unclosed draft genomes, comprising 28 contigs with N50 of 249,915 bp and 251,380 bp for MY1483/09 and Phil1136/12, respectively. B. melitensis $16 \mathrm{M}$ was used to assess the accuracy and effectiveness of the assembled contigs. The total contig sequence lengths for MY1483/09 and Phil1136/12 covered 99.85\% (3,290,051 bp) and 99.92\% $(3,292,385 \mathrm{bp})$ when compared to the reference $16 \mathrm{M}$ genome. The GC content of both isolates was $57.24 \%$, which was similar to that of the $16 \mathrm{M}$ strain (57.22\%).

A total of 3,355 and 3,408 protein encoding genes were predicted and allocated in the chromosomes of the MY1483/09 and Phil1136/12 isolates, respectively, using GeneMarkS v4.10. The predicted functions of 2,920 genes of MY1483/09 (87\% of total predicted genes) and 2,978 genes of Phil1136/12 (87\% of total predicted genes) were obtained by searching against the NR, Swiss-Prot and KEGG databases. Three copies of rRNA in both isolates were identified using rRNAmmer v1.2. A total of 50 and 51 copies of tRNA were predicted using tRNAscan v1.23 in MY1483/09 and Phil1136/12, respectively (Figure $1 \mathrm{~A}$ and $\mathrm{B}$ ).

\section{Phylogenetic analysis}

The whole-genome-based SNP phylogenetic analysis was used to infer the relationships between $B$. melitensis strains collected from diverse geographical locations. We compared the draft genome sequence of the two isolates obtained in this study against 49 strains of B. melitensis (complete genome and draft genome) available in online databases at the time of the analysis. B. melitensis $16 \mathrm{M}$ was used as the reference genome for sequence dataset, containing only the assembled sequences for the SNP discovery using SNPsFinder [17]. As a previous study indicated a close phylogenetic relationship between $B$. melitensis and B. abortus [4], the genomes of two $B$. abortus strains were selected as the outgroup in this analysis. The paralogous genes were removed from the analysis. A comparison of all Brucella strains, including the outgroup strains, with a mismatch cut-off of 8 bp, yielded a total of 13,728 polymorphic nucleotide sites (Additional file 1: Table S1). A phylogenetic tree was 

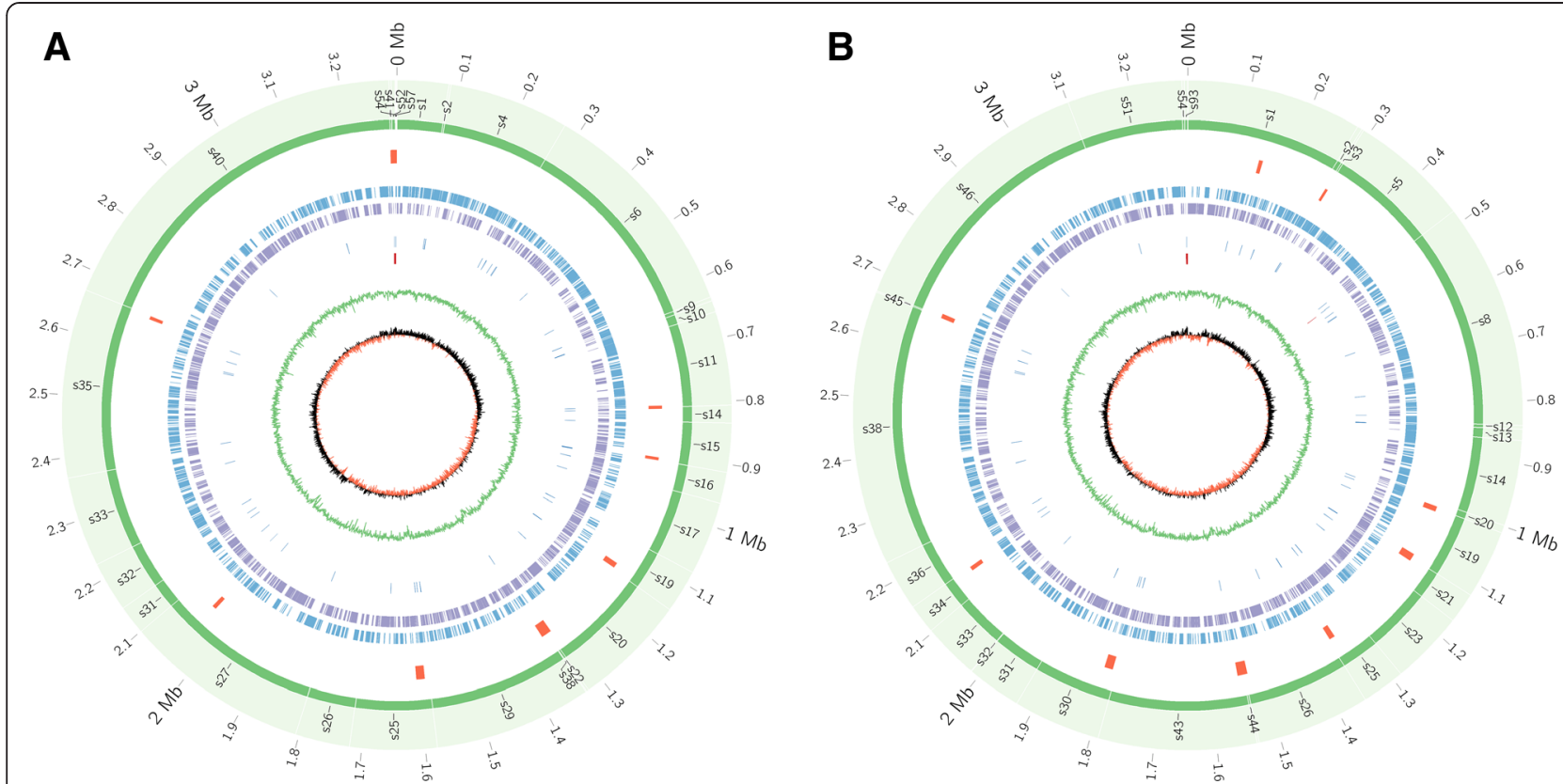

Figure 1 Graphical circular map of the chromosome for (A) MY1483/09 and (B) Phil1136/12. From the outside to the centre: scaffolds (green), genomic islands (red), putative genes in forward (blue), putative genes in reverse (purple), tRNA (dark blue), rRNA (dark red), GC plot (green) and GC skew (orange and black).

reconstructed using the identified SNPs shared among these genomes. The phylogenetic tree, together with the details of the isolates, including the geographical information, the host and the biovar were shown in Figure 2A. All of the B. melitensis strains, except 16M13W and S66, segregated into five major genotypes that corresponded spatially with the potential origins of the isolates. $B$. melitensis Ether (biovar 3) was basal to the B. melitensis clade. B. melitensis $16 \mathrm{M}$ (biovar 1) and 63/9 (biovar 2) were separated after divergence from the Ether strain, demonstrating a similar tree topology that previously reported [4]. Incorporating the tree topology and the age of the $B$. melitensis lineage previously reported [4], we denoted B. melitensis Ether and its related strains as genotype I, $B$. melitensis $63 / 9$ and its related strains as genotype II and $B$. melitensis $16 \mathrm{M}$ and its related strain as genotype $\mathrm{V}$. The $B$. melitensis genotypes III and IV were new genotypes that had not been reported, consisting mainly of recent isolates. Their genetic relationships with other $B$. melitensis strains have therefore not been well described [16]. The overall geographical distribution of the $B$. melitensis genotypes and clades is illustrated in Figure 2B.

Genotype I represented the most basal lineage of the B. melitensis strains. It comprised four out of the $49 \mathrm{~B}$. melitesis strains used in the study. Based on the geographical origin of the strains, most of the genotype I $B$. melitensis strains were isolated from Italy (Ether, F15/06-7 and F5/07-239A) and one isolate, UK31/99 was collected from Egypt. The genotype I strains contained a number of
SNPs, ranging from 2,618 to 2,762 in comparison to the B. melitensis $16 \mathrm{M}$. By conducting the SNP analysis, we identified 936 polymorphic sites that distinguished genotype I strains from other genotypes. A pairwise comparison of the genotype I isolates revealed the presence of 745 intragenotypic SNPs.

Genotype II comprised 30 out of the 49 B. melitensis strains used in the study. It represented the largest $B$. melitensis genotype, with isolates collected from diverse geographical locations, including African, European and Asian countries. It exhibited a ladder-like phylogram, suggesting a possible single introduction of genotype II strains into the region. The genotype II strains possessed SNP differences ranging from 2,187 to 2,521 in comparison to $B$. melitensis $16 \mathrm{M}$. Within genotype II, the isolates further segregated into several clades. A small clade, denoted group $a$, consisted of four B. melitensis strains that emerged from B. melitensis UK3/06 (Cyprus). The genotype $\mathrm{I} b$ strains were collected from distantly related geographical locations, with two of the strains having been collected from European regions, Kosovo and the United Kingdom (UK), one strain from Turkey (Asia) and one strain from Somalia (Africa). A pairwise comparison of the genomes revealed the presence of 295 SNPs within the II $b$ strains. We identified 67 SNPs that were specific to B. melitensis genotype II $b$.

$\mathrm{UK} 29 / 05$, a B. melitensis strain collected from the UK, emerged from genotype II $b$. It occupied the internal node on the phylogenetic tree prior to the emergence of 


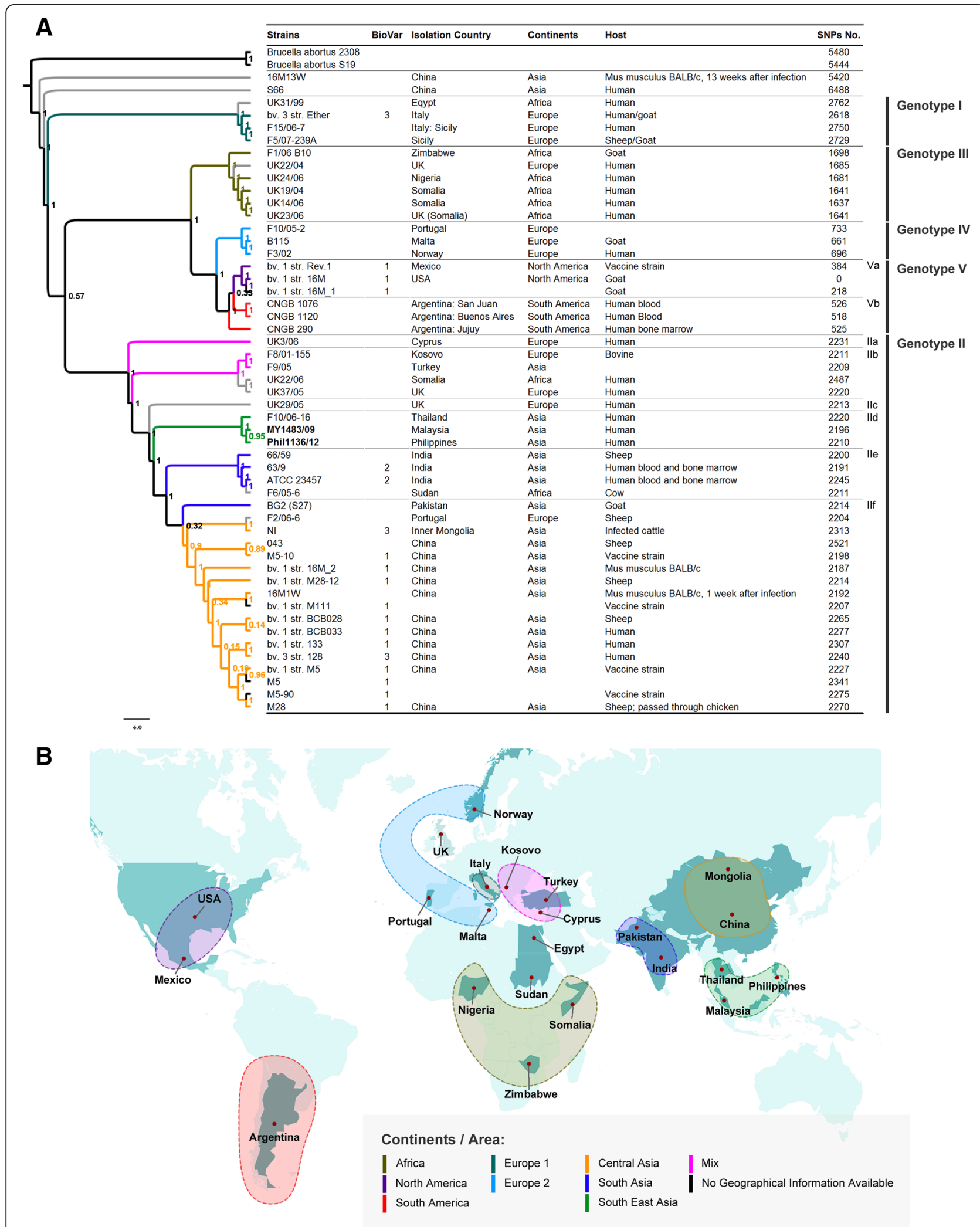

Figure 2 Phylogeography of B. melitensis isolates collected globally. (A) Phylogenetic tree of B. melitensis isolates. The phylogenetic tree was reconstructed using 13,728 polymorphic nucleotide sites and rooted with two B. abortus strains. (B) Geographical distribution of B. melitensis isolates collected globally. 
the Asian B. melitensis strains, herein denoted genotype IIc. All Asian strains (except 16M13W and S66) diverged from genotype IIc. Within the Asian lineage, we described the presence of three geographically distinct clades, SEA, India and China. The two isolates sequenced in the study clustered closely with the Thailand isolate F10/06-16, forming the SEA clade denoted group $d$. Within genotype II $d$, we identified 110 clade-specific SNPs. A pairwise comparison of the SEA clades revealed the presence of 15 SNPs within the SEA B. melitensis population.

The B. melitensis strains continued to diversify and split into two major clades (the Indian and China clades). They shared a common ancestor that was likely to have emerged from genotype IId (SEA clade). The isolates collected from India clustered with $B$. melitensis F6/05-6 collected from Sudan and formed the Indian clade (group $e$ ). Through the SNP analysis, we identified 51 clade-specific SNPs for genotype IIe strains. A pairwise comparison of the Indian lineage revealed the presence of 56 SNPs. The China isolates clustered closely with three strains from Pakistan, Inner Mongolia and Portugal at a branch deep within the China clade, herein denoted group $f$. The laboratory strain, bv1 str. 16 M_2 (China $16 \mathrm{M}$ ), and its derivative, $16 \mathrm{M} 1 \mathrm{~W}$, clustered within group $f$, but not with other B. melitensis $16 \mathrm{M}$ strains (bv1 str. $16 \mathrm{M}$ and bv1 str. 16 M_1) that were sequenced elsewhere. The 16M13W strain (another derivative of China $16 \mathrm{M}$ ) clustered neither in genotype II nor V, but in a group that did not belong to B. melitensis.

Genotypes III, IV and V emerged from the same common ancestral strains and demonstrated strong geographical clustering. Genotype III consisted of strains belonging to the African group, with the exception of UK22/04, which was collected from the UK. The isolates from genotype III had SNPs that numbered from 1,637 to 1,698 in comparison to B. melitensis $16 \mathrm{M}$. Based on the whole-genome SNP analysis, we identified 255 SNPs that were specific to genotype III strains. A pairwise comparison of genotype III strains revealed the presence of 1,388 intragenotypic SNPs. Genotype IV comprised isolates collected from Norway, Portugal and Malta (European clade). We identified 97 clade-specific SNPs that were shared among the genotype IV strains. A pairwise comparison of the genotype IV isolates showed 163 intragenotypic SNPs. Genotype V comprised the American strains collected from Argentina (CNGB1075, CNGB1120 and CNGB290), Mexico (Rev-1) and the USA (16 M strains except China $16 \mathrm{M}$ ). Within genotype V, the strains segregated into two groups, representing those from South America (Argentina) and those from North America (Mexico and USA). We identified 28 specific SNPs for the North American clades. However, we did not observe any specific SNPs for the South American clades.
Our analysis also included genome sequences of all of the vaccine strains of $B$. melitensis used worldwide. Rev-1 is a worldwide vaccine strain $[18,19]$, whereas M5 and M111 are the vaccine strains currently used in China $[8,10]$. Rev-1 belonged to genotype $\mathrm{V}$, grouping closely with $16 \mathrm{M}$ and other isolates collected from Argentina. The M5 and M111 vaccine strains clustered with genotype II group $f$, the China clades. The M5 strain grouped closely with its parental strain, M28.

\section{Discussion}

Anthropogenic activities allow infectious agents to adapt and be disseminated more efficiently to diverse geographical locations by their host. By tracking specific genome modifications, inference of the evolutionary history of the $B$. melitensis strains can be made. The Brucella spp. genome comprises two chromosomes without any additional extrachromosomal genetic elements. The horizontal transfer of genes, a common mechanism contributing to bacterial genome diversity, is limited in Brucella spp., perhaps due to its strict intracellular niche in the host [11]. Genome modifications, including mutations and genes rearrangements, in Brucella spp. therefore, are the exclusive heritable elements during the evolution of the bacteria [20]. The accumulation of mutations or acquisition of genome specific structural changes reflects the microevolution events that drive the evolutionary process, through genetic drift or bacterial adaption, upon introduction into a new geographical region or host. Among the Brucella spp., B. melitensis is the most virulent species for humans. Here, by incorporating a wholegenome SNP-based phylogenetic analysis and identifying the geographical origins of the isolates, we demonstrated a strong phylogenetic link in B. melitensis in relation to its distinctive geographical origins.

The presence of Brucella spp. in the ancient Herculaneum [21] and the high prevalence (17.4\%) of brucellosis in Herculaneum adults [22] demonstrates the prolonged history and continuous circulation of Brucella spp. in the Mediterranean area. A high diversity of B. melitensis strains were collected from countries at the border of the Mediterranean Sea [23-25] and their basal location (genotype 1 ) on the phylogram suggests that $B$. melitensis could have originated from this region. This is further supported by the recent discovery of a 700 year-old $B$. melitensis genotype I strain from Sardinia [26]. Ancient and Old World transcontinental trading was an early form of globalisation that allowed the exchange of goods among the continents. The infectious agents that attached to humans or goods, such as livestock and its derivatives spread in a parallel manner [27]. Based on the phylogenetic tree drawn in this study using whole-genome SNPs, it was shown that the $B$. melitensis genotype I gave rise to two major branches, representing $B$. melitensis strains collected 
from the East (genotype II) and West (genotype III/IV/V). The strains were likely to have been distributed along the major ancient trade routes, e.g., the ancient Silk Road, the Trans-Eurasia exchange and the Columbian exchange. The transcontinental spread of $B$. melitensis could therefore have marked the beginning of the diversification of the $B$. melitensis strains. The global spread of $B$. melitensis may have occurred following these ancient trading routes, perhaps through infected goat and sheep or their milk derivatives.

B. melitensis genotype II had the most diverse geographical distribution after diverging from genotype I. Following the phylogenetic ladder pattern, the distribution of genotype II strains expanded from West (Cyprus) to East (China). Its ladder-like tree topology suggests that the genotype II strains descended from a single common ancestor that originated from the Mediterranean area (Cyprus origin?) to form the Asian clades, as almost all Asian strains of B. melitensis belonged to this group. It is noted that B. melitensis BG2 (S27), isolated from Pakistan, occupied the ancestral node of group $f$ (China clade), suggesting that the $B$. melitensis strain currently circulating in China may has a Pakistani origin.

Genotypes III, IV and V of B. melitensis demonstrated a more restricted distribution. They shared a common ancestor, which most likely diversified and spread towards the southern region of the Mediterranean, and formed genotype III (African lineage). The strains that spread northwards could have further diversified and adapted geographically forming genotype IV (European lineage) and genotype V (American lineage). Compared with the American region, genotype IV of B. melitensis strains were more geographically diverse (Portugal, Malta and Norway). Unlike Portugal and Malta, Norway is officially a brucellosis-free country [28], with no $B$. melitensis having been detected in sheep or goat herds to date [29]. Therefore, the Norway strain, F3/02, most likely represents an imported case that probably originated in the northern part of the Mediterranean region. There were limited numbers of sequences available for analysis from this region. Thus, we cannot rule out the sampling bias that may have affected the assignment of genotype IV to the northern Mediterranean area.

The genotype $\mathrm{V}$ strains were most probably introduced from Europe into the American continent by infected animals several hundred years ago, where $B$. melitensis could have continued to diversify into two geographically specific sub-lineages, genotype Va (North American lineage) and $\mathrm{Vb}$ (South American lineage). Nevertheless, only two strains (three genome sequences) and three strains were used to designate genotypes $\mathrm{Va}$ and $\mathrm{Vb}$, respectively. The two genotype Va strains, $16 \mathrm{M}$ (USA origin) [30] and Rev-1 (Mexico origin; vaccine strain developed in USA laboratories) [31,32], however, may not reflect the currently circulating B. melitensis strain in the North American region. Similarly, all three $B$. melitensis strains used for the designation of genotype $\mathrm{Vb}$ were isolated from Argentina. Although the origin of the Argentinian strains in this study (native or imported) remains inconclusive, the continuing circulation of $B$. melitensis in Mexico and Argentina, where $B$. melitensis is the principle cause of brucellosis [33], suggests the existence of native $B$. melitensis in the American continent. With the current data, we demonstrated that genotype $\mathrm{V}$ of $B$. melitensis was only detected in the American region, supporting the geographical specificity of genotype V. More field $B$. melitensis strains from diverse geographical regions of the American continent, however, are needed to conclude the designation of genotype $\mathrm{V}$.

The SEA region is believed to have been free of $B$. melitensis until recently, similar to Australia, New Zealand, Northern Europe, the US and Canada [34,35]. Recent epidemiological studies of livestock and B. melitensis associated with human brucellosis, however, have suggested that the bacteria could have been transmitted and maintained unknowingly in the goat and sheep populations [36-41]. These SEA B. melitensis strains have not been well studied. Genetic markers, such as IS711 used for species characterisation in previous studies, are not sufficient to delineate intraspecies relationships of $B$. melitensis $[40,41]$. Here, using a comprehensive whole-genome-based SNP approach, the two $B$. melitensis strains obtained in this study clustered closely with the Thailand B. melitensis strains (F10/06-16) and formed a new SEA clade. Deciphering the origin and source of the SEA B. melitensis strains is highly challenging, owing to the existence of both local and imported goat breeds in SEA countries. We identified 110 SEA clade-specific SNPs that differentiated the SEA clade from other global $B$. melitensis strains, suggesting a common ancestor of the SEA B. melitensis strains in this region. A previous study suggested that the persistence of B. melitensis in wildlife could serve as a reservoir for endemic outbreaks [42]. The high genetic similarity (15 polymorphic SNPs) of the three SEA B. melitensis strains, however, suggests that the spread of SEA strains was relatively recent. It is therefore unlikely that brucellosis was caused by autochthonous transmission from local breeds in the wild. Moreover, Malaysia, the Philippines and Thailand are not major exporters of goats and sheep in the region. Therefore, it is plausible that the $B$. melitensis in this region was introduced from a common source through the importation of goats and sheep from elsewhere. The bacterial strains became established and adapted to the local environment, leading to the spread of $B$. melitensis to the local goat and sheep populations.

In view of the persistent infection caused by the vaccine strains in animals [43,44], their possible 
involvement in human infection is worth investigating. Data concerning the endemic strains and the use of vaccines in local animal husbandry, however, have not been well documented. It is possible that the vaccine strains used in animals could intentionally be used to cause harm to humans. There are several veterinary vaccine Brucella spp. strains currently in use. We showed here, by using a wholegenome SNP-based phylogenetic tree, that we were able to resolve and discriminate between the vaccine strains and the endemic strains. This is particularly important to help identify possible sources of infection and to monitor the potential misuse of live attenuated vaccines.

Through the whole-genome SNP analysis, two previously assigned B. melitensis strains, S66 and 16M13W, segregated into a group that did not belong to B. melitensis. S66 was previously reported as a $B$. melitensis sequence type 8 strain [45], while $16 \mathrm{M} 13 \mathrm{~W}$ is the 13 -week mice-adapted strain of China $16 \mathrm{M}$ B. melitensis [9]. Clustering of the $16 \mathrm{M} 13 \mathrm{~W}$ strain into a different group from its parental strain suggests that it may not have originated from China $16 \mathrm{M}$. Although both China $16 \mathrm{M}$ and $16 \mathrm{M} 1 \mathrm{~W}$ were identified as B. melitensis strains, they were not grouped with other previously designated $16 \mathrm{M}$ strains (bv1 str. $16 \mathrm{M}$ and bv1 str. 16 M_1). In contrast, they grouped with other $B$. melitensis strains collected from China (genotype II), raising concern that the China $16 \mathrm{M}$ may not be the same $16 \mathrm{M}$ strain used worldwide.

To date, serological tests, such as the agglutination test, and isolation of bacteria from blood cultures remain the gold standard for the diagnosis of human brucellosis. The interpretation of the results from such assays, however can be difficult due to the serological cross-reaction of Brucella spp. with other bacteria [46,47]. In addition, differential identification of closely related Brucella spp. is not possible using a single serological test. The PCR-based genome sequence amplification method is a good alternative, as it allows for rapid identification of the bacteria. Differential identification of highly similar Brucella spp., however is only achievable with multiple gene sequence analyses, such as MLS and MLVA. With the limited candidate genes used for MLS and MLVA typing, the intraspecies relationships of closely related species of $B$. melitensis are difficult to resolve. In comparison to these subtyping approaches, the whole-genome SNP-based phylogenetic analysis presented here provides better resolution power to resolve the genetic relationships between the $B$. melitensis species.

\section{Conclusion}

In summary, we have demonstrated that SNPs retrieved from the $B$. melitensis draft genomes were sufficient to resolve the interspecies relationships within $B$. melitensis strains. Our findings obtained using the genome SNP analyses revealed potential $B$. melitensis endemicity to the SEA region. The B. melitensis phylogeny presented here describes the relationships between the global $B$. melitensis strains and their evolutionary history. The whole-genome SNP-based approach provides an effective means of determining the native geographical origin of the pathogen and could serve as the basis for the reconstruction of the history of the global spread of $B$. melitensis at a finer scale. In addition, we have discriminated between the vaccine and endemic strains using the current whole-genome SNP approach. This will allow the rapid identification of pathogens and sensitive epidemiological follow-up in cases of release with intention to cause harm.

\section{Methods}

\section{Bacterial strains}

The Brucella strains MY1483/09 (GenBank: JN571438) [37] and Phil1136/12 were previously isolated from brucellosis patients admitted to hospitals in Kuala Lumpur. One patient had consumed unpasteurised cow's milk in Malaysia (MY1483/09), while the other had drunk unpasteurised goat's milk in the Philippines (Phil1136/12).

\section{Genomic DNA sample preparation}

The bacteria were cultured in Brucella broth and incubated at $37^{\circ} \mathrm{C}$ for 72 hours. Genomic DNA was extracted from $5 \mathrm{~mL}$ of culture using the QIAamp DNA Mini Kit (Qiagen, Germany). All sample handling was performed in a BSL-3 biocontainment laboratory at the Tropical Infectious Diseases Research and Educational Centre (TIDREC), University of Malaya. The genomic DNA concentration was quantified using the Qubit $^{\circ}$ dsDNA BR Assay Kit (Invitrogen, Life Technologies, USA).

\section{Full genome sequencing}

The genome library preparation was performed by using the Ion Xpress ${ }^{\mathrm{TM}}$ Plus Fragment Library Kit (Life Technologies, USA) following the manufacturer's protocol, 4471989 Rev E. The sheared DNA was separated using a 2\% agarose gel (Promega, USA) and fragments that corresponded to $200 \mathrm{bp}$ of a sequencing run (approximately $330 \mathrm{bp}$ ) were manually excised. The DNA was purified using the QIAquick Gel Extraction Kit (Qiagen, Germany). The library quality and concentration were assessed and determined using the 2100 Bioanalyzer (Agilent, USA) and High Sensitivity DNA Kit (Agilent, USA). The sequencing template was prepared using the Ion OneTouch $^{\mathrm{TM}} 200$ Template Kit V2 DL (Life Technologies, USA) according to the manufacturer's protocol. The Ion Sphere Particles were enriched using Dynabeads MyOne Streptavidin C1 beads (Invitrogen, Life Technologies, USA). The efficiency of the enrichment process was assessed using the Ion Sphere Quality Control Kit (Life Technologies, USA). Genome sequencing was undertaken using the Ion Torrent PGM sequencer (Life Technologies, USA). 
Table 1 Brucella spp. strains used in the analysis in this study

\begin{tabular}{|c|c|c|c|}
\hline No & Organism & Abbreviation & GenBank Assembly ID \\
\hline 1 & Brucella melitensis 043 & 043 & GCA_000331655.1 \\
\hline 2 & Brucella melitensis $16 \mathrm{M} 13 \mathrm{~W}$ & $16 \mathrm{M} 13 \mathrm{~W}$ & GCA_000250835.2 \\
\hline 3 & Brucella melitensis $16 \mathrm{M} 1 \mathrm{~W}$ & $16 \mathrm{M} 1 \mathrm{~W}$ & GCA_000250815.2 \\
\hline 4 & Brucella melitensis 66/59 & $66 / 59$ & GCA_000366865.1 \\
\hline 5 & Brucella melitensis ATCC 23457 & ATCC 23457 & GCA_000022625.1 \\
\hline 6 & Brucella melitensis B115 & B115 & GCA_000365865.1 \\
\hline 7 & Brucella melitensis BG2 (S27) & BG2 (S27) & GCA_000370625.1 \\
\hline 8 & Brucella melitensis bv. 1 str. 133 & bv. 1 str. 133 & GCA_000298595.1 \\
\hline 9 & Brucella melitensis bv. 1 str. $16 \mathrm{M}$ & bv. 1 str. $16 \mathrm{M}$ & GCA_000007125.1 \\
\hline 10 & Brucella melitensis bv. 1 str. 16M_1 & bv. 1 str. 16M_1 & GCA_000160295.1 \\
\hline 11 & Brucella melitensis bv. 1 str. 16M_2 & bv. 1 str. 16M_2 & GCA_000250795.2 \\
\hline 12 & Brucella melitensis bv. 1 str. BCB028 & bv. 1 str. BCB028 & GCA_000292165.2 \\
\hline 13 & Brucella melitensis bv. 1 str. BCB033 & bv. 1 str. BCB033 & GCA_000292085.2 \\
\hline 14 & Brucella melitensis bv. 1 str. M111 & bv. 1 str. M111 & GCA_000209615.1 \\
\hline 15 & Brucella melitensis bv. 1 str. M28-12 & bv. 1 str. M28-12 & GCA_000209595.1 \\
\hline 16 & Brucella melitensis bv. 1 str. M5 & bv. 1 str. M5 & GCA_000348645.1 \\
\hline 17 & Brucella melitensis bv. 1 str. Rev.1 & bv. 1 str. Rev. 1 & GCA_000158695.1 \\
\hline 18 & Brucella melitensis bv. 2 str. $63 / 9$ & $63 / 9$ & GCA_000182235.1 \\
\hline 19 & Brucella melitensis bv. 3 str. 128 & bv. 3 str. 128 & GCA_000298615.1 \\
\hline 20 & Brucella melitensis bv. 3 str. Ether & bv. 3 str. Ether & GCA_000158735.1 \\
\hline 21 & Brucella melitensis CNGB 1076 & CNGB 1076 & GCA_000366625.1 \\
\hline 22 & Brucella melitensis CNGB 1120 & CNGB 1120 & GCA_000366885.1 \\
\hline 23 & Brucella melitensis CNGB 290 & CNGB 290 & GCA_000366905.1 \\
\hline 24 & Brucella melitensis F1/06 B10 & F1/06 B10 & GCA_000370645.1 \\
\hline 25 & Brucella melitensis F10/05-2 & $\mathrm{F} 10 / 05-2$ & GCA_000366925.1 \\
\hline 26 & Brucella melitensis F10/06-16 & F10/06-16 & GCA_000370665.1 \\
\hline 27 & Brucella melitensis F15/06-7 & F15/06-7 & GCA_000365845.1 \\
\hline 28 & Brucella melitensis F2/06-6 & $F 2 / 06-6$ & GCA_000366945.1 \\
\hline 29 & Brucella melitensis F3/02 & $\mathrm{F} 3 / 02$ & GCA_000366965.1 \\
\hline 30 & Brucella melitensis F5/07-239A & $\mathrm{F} 5 / 07-239 \mathrm{~A}$ & GCA_000366985.1 \\
\hline 31 & Brucella melitensis F6/05-6 & $F 6 / 05-6$ & GCA_000367005.1 \\
\hline 32 & Brucella melitensis F8/01-155 & F8/01-155 & GCA_000370685.1 \\
\hline 33 & Brucella melitensis F9/05 & $\mathrm{F} 9 / 05$ & GCA_000370705.1 \\
\hline 34 & Brucella melitensis M28 & $M 28$ & GCA_000192725.1 \\
\hline 35 & Brucella melitensis M5 & M5 & GCA_000209575.1 \\
\hline 36 & Brucella melitensis M5-10 & M5-10 & GCA_000292065.1 \\
\hline 37 & Brucella melitensis M5-90 & M5-90 & GCA_000192885.1 \\
\hline 38 & Brucella melitensis MY1483/09 & MY1483/09 & In this study \\
\hline 39 & Brucella melitensis $\mathrm{NI}$ & $\mathrm{NI}$ & GCA_000227645.1 \\
\hline 40 & Brucella melitensis Phil1136/12 & Phil1136/12 & In this study \\
\hline 41 & Brucella melitensis S66 & S66 & GCA_000250775.2 \\
\hline 42 & Brucella melitensis UK14/06 & UK14/06 & GCA_000370725.1 \\
\hline 43 & Brucella melitensis UK19/04 & UK19/04 & GCA_000367045.1 \\
\hline 44 & Brucella melitensis UK22/04 & UK22/04 & GCA 000370745.1 \\
\hline
\end{tabular}


Table 1 Brucella spp. strains used in the analysis in this study (Continued)

\begin{tabular}{llll}
\hline 45 & Brucella melitensis UK22/06 & UK22/06 & GCA_000366585.1 \\
46 & Brucella melitensis UK23/06 & UK23/06 & GCA_000370765.1 \\
47 & Brucella melitensis Uk24/06 & UK24/06 & GCA_000370785.1 \\
48 & Brucella melitensis UK29/05 & UK29/05 & GCA_000370805.1 \\
49 & Brucella melitensis UK3/06 & UK3/06 & GCA_000370825.1 \\
50 & Brucella melitensis UK31/99 & UK31/99 & GCA_000370845.1 \\
51 & Brucella melitensis UK37/05 & UK37/05 & GCA_000370865.1 \\
52 & Outgroup Brucella abortus 2308 & Brucella abortus 2308 & GCA_000054005.1 \\
53 & Outgroup Brucella abortus S19 & Brucella abortus S19 & GCA_000018725.1 \\
\hline
\end{tabular}

\section{Genome assembly and annotation}

The Ion Torrent generated reads were assembled against B. melitensis $16 \mathrm{M}$ (GeneBank: NC003317 and GenBank: NC003318) using MIRA 3, as implemented in Torrent Suite V4.0.2. Assemblies of the two isolates were ordered and aligned based on that of $B$. melitensis $16 \mathrm{M}$ by Mauve v2.3.1 [48]. Potential mis-assembly points identified from the alignment were verified by PCR and DNA sequencing using capillary electrophoresis. Scaffolds with mis-assembly points were manually broken and joined using Gap5 v1.2.14 [49]. The putative tRNA and rRNA were identified by tRNAscan v1.23 [50] and rRNAmmer v1.2 [51], respectively. The protein coding genes were predicted by GeneMarkS v4.10 [52]. The putative identities of these genes were annotated by running the Basic Local Alignment Search Tool (BLAST) [53] against the NCBI non-redundant, Swiss-Prot [54] and Kyoto Encyclopaedia of Genes and Genomes (KEGG) [55] databases.

\section{SNP discovery and phylogenetic construction}

SNP divergence of all 53 genomes (Table 1, 2 draft genomes in this study, 5 complete and 44 drafts, publicly available genomes of B. melitensis and 2 genomes of B. abortus as outgroups) was discovered using the web-based programme SNPs Finder [17]. The SNPs were evaluated in homologous regions of $600 \mathrm{bp}$ that shared sequence similarity of at least $99 \%$. The repetitive/paralogous sequences in the alignment were eliminated by the algorithm in the SNPs Finder software [17] to reduce false positive SNP identification. The deduced SNP set was filtered by eliminating the SNPs that were close to each other at a distance of less than $8 \mathrm{bp}$, as previously reported [4], using in house scripts.

The phylogenetic relationships of the 53 genomes were constructed using MrBayes v3.2.1 [56]. Bayesian MCMC analysis was conducted by sampling across the entire general time reversible (GTR) model space. One million generations were run with a sampling frequency of 500 and diagnostics were calculated for every 5000 generations.
A burn-in setting of $25 \%$ was used to discard the first 500 trees. Convergence was assessed manually with the standard deviation of split frequencies falling below 0.01. There was no obvious trend for the plot of the generation versus the log probability of the data (the log likelihood values) and the potential scale reduction factor (PSRF) was reasonably close to 1.0 for all parameters.

\section{Supporting data}

The genome sequences generated in this study are available from the European Nucleotide Archive with accession numbers PRJEB7499 (http://www.ebi.ac.uk/ena/ data/view/PRJEB7499) and PRJEB7504 (http://www.ebi.ac. uk/ena/data/view/PRJEB7504) for MY1483/09 and Phil1136/ 12 , respectively.

\section{Additional file}

Additional file 1: Table S1. List of 13,728 polymorphic nucleotide sites of Brucella spp. that used for phylogenetic tree reconstruction.

\section{Competing interests}

The authors declare that they have no competing interests.

\section{Authors' contributions}

KKT performed the experiments, analysed and interpreted the data and wrote the manuscript. YCT analysed and interpreted the data, prepared figures and assisted in manuscript writing. KWL, WYY and $\mathrm{CCH}$ analysed and interpreted the data and assisted in manuscript writing. LYC, SSN and FLJ performed the bacterial cultivation and DNA extraction. MNMI participated in the genome analysis. SAB conceived and designed the study, coordinated the experiments, analysed and interpreted the data and wrote the manuscript. All authors have read and approved the final manuscript.

\section{Acknowledgements}

We thank Miss Khor Sheau-Sean for her help in genome data analysis. We thank the following institutes for the Brucella genome sequences that were used in this study: the Ministry of Science and Technology of the People's Republic of China (GenBank: GCA 000331655), the Academy of Military Medical Science, China (GenBank: GCA 000250835, GCA_000250815, GCA 000250795, GCA 000250775), the Virginia Bioinformatics Institute (GenBank: GCA 000022625, GCA 000018725), the Beijing Institute of Disease Control and Prevention (GenBank: GCA 000298595, GCA 000292165, GCA 000292085, GCA 000298615, GCA 000292065), Integrated Genomics (GenBank: GCA 
000007125), the Chinese Academy of Science (GenBank: GCA 000209615, GCA 000209595, GCA 000209575), the Chinese Centre for Disease Control and Prevention (GenBank: GCA 000348645), the Chinese Academy of Agricultural Sciences (GenBank: GCA 000192725, GCA 000192885), the Chinese Agricultural University (GenBank: GCA 000227645), Oak Ridge National Laboratory (GenBank: GCA 000054005) and the Broad Institute (GenBank: GCA 000366865, GCA 000365865, GCA 000370625, GCA 000160295, GCA 000158695, GCA 000182235, GCA 000158735, GCA 000366625, GCA 000366885, GCA 000366905, GCA 000370645, GCA 000366925, GCA 000370665, GCA 000365845 , GCA 000366945 , GCA 000366965 , GCA 000366985 , GCA 000367005 , GCA 000370685, GCA 000370705, GCA 000370725, GCA 000367045, GCA 000370745, GCA 000366585, GCA 000370765, GCA 000370785, GCA 000370805, GCA 000370825, GCA 000370845, GCA 000370865). The research leading to these results received funding from the University of Malaya High Impact Research (HIR) grant scheme, with agreement numbers H-20001-00-E000011 and E000013-20001. The sequence analyses were made possible using bioinformatics infrastructure funded through the Ministry of Science, Technology, and Innovation, (Malaysia Genome Institute initiative grant 07-05-MGI-GMB015), Malaysia.

\section{Author details}

${ }^{1}$ Tropical Infectious Diseases Research and Education Centre (TIDREC), University of Malaya, 50603 Kuala Lumpur, Malaysia. ${ }^{2}$ Department of Medical Microbiology, Faculty of Medicine, University of Malaya, 50603 Kuala Lumpur, Malaysia. ${ }^{3}$ Codon Genomics S/B, No 26, Jalan Dutamas 7, Taman Dutamas, Balakong, 43200 Seri Kembangan, Selangor, Malaysia. ${ }^{4}$ Malaysia Genome Institute, Ministry of Science, Technology and Innovation, Jalan Bangi, 43000 Kajang, Selangor, Malaysia.

\section{Received: 5 October 2014 Accepted: 29 January 2015}

\section{Published online: 18 February 2015}

\section{References}

1. Song $L, A$ Ahn $S$, Walt DR. Detecting biological warfare agents. Emerg Infect Dis. 2005;11(10):1629-32.

2. Franz DR, Parrott CD, Takafuji ET. The US biological warfare and biological defense programs. In: Sidell FR, Takafuji ET, Franz DR, editors. Medical Aspects of Chemical and Biological Warfare. United of States: Office of The Surgeon General (Army; 1997. p. 425-36.

3. Kaufmann AF, Meltzer MI, Schmid GP. The economic impact of a bioterrorist attack: are prevention and postattack intervention programs justifiable? Emerg Infect Dis. 1997;3(2):83-94.

4. Foster JT, Beckstrom-Sternberg SM, Pearson T, Beckstrom-Sternberg JS, Chain PS, Roberto FF, et al. Whole-genome-based phylogeny and divergence of the genus Brucella. J Bacteriol. 2009;191(8):2864-70.

5. Read TD, Peterson SN, Tourasse N, Baillie LW, Paulsen IT, Nelson KE, et al. The genome sequence of Bacillus anthracis Ames and comparison to closely related bacteria. Nature. 2003;423(6935):81-6.

6. Foster JT, Price LB, Beckstrom-Sternberg SM, Pearson T, Brown WD, Kiesling DM, et al. Genotyping of Brucella species using clade specific SNPs. BMC Microbiol. 2012:12:110.

7. Liu W, Jing Z, Ou Q, Cui B, He Y, Wu Q. Complete genome sequence of Brucella melitensis biovar 3 strain $\mathrm{NI}$, isolated from an aborted bovine fetus. J Bacteriol. 2012;194(22):6321.

8. Wang F, Hu S, Gao Y, Qiao Z, Liu W, Bu Z. Complete genome sequences of Brucella melitensis strains M28 and M5-90, with different virulence backgrounds. J Bacteriol. 2011;193(11):2904-5.

9. Ke $Y$, Yuan $X$, Wang $Y$, Bai $Y, X u J$, Song $H$, et al. Genome sequences of Brucella melitensis $16 \mathrm{M}$ and its two derivatives $16 \mathrm{M} 1 \mathrm{w}$ and $16 \mathrm{M} 13 \mathrm{w}$ which evolved in vivo. J Bacteriol. 2012;194(19):5489.

10. Ding J, Pan $Y$, Jiang $H$, Cheng J, Liu T, Qin N, et al. Whole genome sequences of four Brucella strains. J Bacteriol. 2011;193(14):3674-5.

11. Wattam AR, Williams KP, Snyder EE, Almeida Jr NF, Shukla M, Dickerman AW, et al. Analysis of ten Brucella genomes reveals evidence for horizontal gene transfer despite a preferred intracellular lifestyle. J Bacteriol. 2009;191(11):3569-79.

12. Wattam AR, Foster JT, Mane SP, Beckstrom-Sternberg SM, Beckstrom-Sternberg $J M$, Dickerman AW, et al. Comparative phylogenomics and evolution of the Brucellae reveal a path to virulence. J Bacteriol. 2014;196(5):920-30.

13. Wattam AR, Inzana TJ, Williams KP, Mane SP, Shukla M, Almeida NF, et al. Comparative genomics of early-diverging Brucella strains reveals a novel lipopolysaccharide biosynthesis pathway. mBio. 2012;3(5):e00246-00211.
14. Girault G, Blouin Y, Vergnaud G, Derzelle S. High-throughput sequencing of Bacillus anthracis in France: investigating genome diversity and population structure using whole-genome SNP discovery. BMC Genomics. 2014;15(1):288

15. Chen PE, Willner KM, Butani A, Dorsey S, George M, Stewart A, et al. Rapid identification of genetic modifications in Bacillus anthracis using whole genome draft sequences generated by 454 pyrosequencing. PLoS One. 2010:5(8):e12397.

16. Wattam AR, Abraham D, Dalay O, Disz TL, Driscoll T, Gabbard JL, et al. PATRIC, the bacterial bioinformatics database and analysis resource. Nucleic Acids Res. 2014;42(Database issue):D581-91.

17. Song J, Xu Y, White S, Miller KW, Wolinsky M. SNPsFinder-a web-based application for genome-wide discovery of single nucleotide polymorphisms in microbial genomes. Bioinformatics. 2005;21(9):2083-4.

18. Elberg SS, Faunce K. Immunization Against Brucella Infection VI. Brucella melitensis immunity conferred on goats by a nondependent mutant from a streptomycin-dependent mutant strain of Brucella melitensis. J Bacteriol. 1957;73(2):211-7

19. Cloeckaert A, Grayon M, Grépinet O. Identification of Brucella melitensis vaccine strain Rev. 1 by PCR-RFLP based on a mutation in the rps $L$ gene. Vaccine. 2002;20(19):2546-50.

20. Moreno E. Genome evolution within the alpha proteobacteria: why do some bacteria not possess plasmids and others exhibit more than one different chromosome? FEMS Microbiol Rev. 1998;22(4):255-75.

21. Capasso L. Bacteria in two-millennia-old cheese, and related epizoonoses in Roman populations. J Infect. 2002;45(2):122-7.

22. Capasso L. Brucellosis at Herculaneum (79 AD). Int J Osteoarchaeol. 1999;9(5):277-88.

23. Valdezate S, Navarro A, Villalon P, Carrasco G, Saez-Nieto JA. Epidemiological and phylogenetic analysis of Spanish human Brucella melitensis strains by multiple-locus variable-number tandem-repeat typing, hypervariable octameric oligonucleotide fingerprinting, and rpoB typing. J Clin Microbiol. 2010;48(8):2734-40.

24. Sayan M, Yumuk Z, Bilenoglu O, Erdenlig S, Willke A. Genotyping of Brucella melitensis by rpoB gene analysis and re-evaluation of conventional serotyping method. Jpn J Infect Dis. 2009;62(2):160-3.

25. Marianelli C, Ciuchini F, Tarantino M, Pasquali P, Adone R. Molecular characterization of the $r p o B$ gene in Brucella species: new potential molecular markers for genotyping. Microb Infect/Institut Pasteur. 2006;8(3):860-5.

26. Kay GL, Sergeant MJ, Giuffra V, Bandiera P, Milanese M, Bramanti B, et al. Recovery of a medieval Brucella melitensis genome using shotgun metagenomics. mBio. 2014;5(4):e01337-01314.

27. Morelli G, Song Y, Mazzoni CJ, Eppinger M, Roumagnac P, Wagner DM, et al. Yersinia pestis genome sequencing identifies patterns of global phylogenetic diversity. Nat Genet. 2010;42(12):1140-3.

28. Godfroid J, Kasbohrer A. Brucellosis in the European Union and Norway at the turn of the twenty-first century. Vet Microbiol. 2002;90(1-4):135-45.

29. Kampen AH, Bakken EH, Jore S, Klevar S. The surveillance programme for Brucella melitensis in small ruminants in Norway 2013. In: Surveillance programmes for terrestrial and aquatic animals in Norway Annual report 2013. Oslo: Norwegian Veterinary Institute; 2014.

30. Meyer ME, Morgan W. Designation of neotype strains and of biotype reference strains for species of the genus Brucella Meyer and Shaw. Int J Syst Bacteriol. 1973;23(2):135-41.

31. Verger J-M, Grimont F, Grimont PA, Grayon M. Brucella, a monospecific genus as shown by deoxyribonucleic acid hybridization. Int J Syst Bacteriol. 1985;35(3):292-5

32. Herzberg M, Elberg S. Immunization against Brucella infection I.: Isolation and Characterization of a Streptomycin-Dependent Mutant1. J Bacteriol. 1953:66(5):585

33. Lucero NE, Ayala SM, Escobar Gl, Jacob NR. Brucella isolated in humans and animals in Latin America from 1968 to 2006. Epidemiol Infect. 2008;136(4):496-503.

34. Olsen S, Bellaire B. Brucella. In McVey DS, Kennedy M, Chengappa MM, editors. Veterinary Microbiology, 3 edn. United of States: Wiley; 2013. P. 127-133

35. Robinson A. Guidelines for coordinated human and animal brucellosis surveillance. 2003.

36. Wongphruksasoong V, Santayakorn S, Sitthi W, Chuxnum T, Pipatjaturong N, Kunthu A, et al. An Outbreaks of Brucella melitensis among goat farmers in Thailand, December 2009. Outbreak, Surveillance and Investigation Reports. 2012;5(1):14-21. 
37. Sam IC, Karunakaran R, Kamarulzaman A, Ponnampalavanar S, Syed Omar SF, Ng KP, et al. A large exposure to Brucella melitensis in a diagnostic laboratory. J Hosp Infect. 2012;80(4):321-5.

38. Manosuthi W, Thummakul T, Vibhagool A, Vorachit M, Malathum K. Case report: Brucellosis: a re-emerging disease in Thailand. Southeast Asian J Trop Med Public Health. 2004;35(1):109-12.

39. Jama'ayah MZ, Heu JY, Norazah A. Seroprevalance of brucellosis among suspected cases in Malaysia. Malays J Pathol. 2011;33(1):31-4.

40. Bamaiyi PH, Hassan L, Khairani-Bejo S, Zainal Abidin M, Ramlan M, Krishnan $\mathrm{N}$, et al. Isolation and molecular characterization of Brucella melitensis from seropositive goats in Peninsula Malaysia. Trop Biomed. 2012;29(4):513-8.

41. Al-Garadi MA, Khairani-Bejo S, Zunita Z, Omar A. Isolation and identification of Brucella melitensis in goats. J Anim Vet Adv. 2011:10(8):972-9.

42. Mick V, Le Carrou G, Corde Y, Game Y, Jay M, Garin-Bastuji B. Brucella melitensis in France: persistence in wildlife and probable spillover from Alpine ibex to domestic animals. PLoS One. 2014;9(4):e94168.

43. Corner LA, Alton GG. Persistence of Brucella abortus strain 19 infection in adult cattle vaccinated with reduced doses. Res Vet Sci. 1981;31(3):342-4.

44. Meyer ME, Nelson CJ. Persistence of Brucella abortus, strain 19 infection in immunized cattle. Proc Annu Meet US Anim Health Assoc. 1969;73:159-65.

45. Ke Y, Yuan X, Zhen Q, Wang Y, Li T, Sun Y, et al. Genome sequence of Brucella melitensis S66, an isolate of sequence type 8, prevalent in China. J Bacteriol. 2012;194(19):5451.

46. Corbel MJ, Stuart FA, Brewer RA. Observations on serological cross-reactions between smooth Brucella species and organisms of other genera. Dev Biol Stand. 1984:56:341-8

47. Francis E, Evans AC: Agglutination, cross-agglutination and agglutinin absorption in tularaemia. Public Health Rep. 1896-1970. 1926;1273-1295

48. Darling AC, Mau B, Blattner FR, Perna NT. Mauve: multiple alignment of conserved genomic sequence with rearrangements. Genome Res. 2004;14(7):1394-403.

49. Bonfield JK, Whitwham A. Gap5-editing the billion fragment sequence assembly. Bioinformatics. 2010;26(14):1699-703.

50. Schattner P, Brooks AN, Lowe TM. The tRNAscan-SE, snoscan and snoGPS web servers for the detection of tRNAs and snoRNAs. Nucleic Acids Res. 2005;33(Web Server issue):W686-9.

51. Lagesen K, Hallin P, Rodland EA, Staerfeldt HH, Rognes T, Ussery DW. RNAmmer: consistent and rapid annotation of ribosomal RNA genes. Nucleic Acids Res. 2007:35(9):3100-8.

52. Besemer J, Lomsadze A, Borodovsky M. GeneMarkS: a self-training method for prediction of gene starts in microbial genomes. Implications for finding sequence motifs in regulatory regions. Nucleic Acids Res. 2001;29(12):2607-18.

53. Altschul SF, Gish W, Miller W, Myers EW, Lipman DJ. Basic local alignment search tool. J Mol Biol. 1990:215(3):403-10.

54. Bairoch A, Apweiler R. The SWISS-PROT protein sequence database and its supplement TrEMBL in 2000. Nucleic Acids Res. 2000;28(1):45-8.

55. Ogata H, Goto S, Sato K, Fujibuchi W, Bono H, Kanehisa M. KEGG: Kyoto Encyclopedia of Genes and Genomes. Nucleic Acids Res. 1999;27(1):29-34.

56. Huelsenbeck JP, Ronquist F. MRBAYES: Bayesian inference of phylogenetic trees. Bioinformatics. 2001:17(8):754-5.

\section{Submit your next manuscript to BioMed Central and take full advantage of:}

- Convenient online submission

- Thorough peer review

- No space constraints or color figure charges

- Immediate publication on acceptance

- Inclusion in PubMed, CAS, Scopus and Google Scholar

- Research which is freely available for redistribution 